\title{
Wie man eine erfolgreiche Praxis gestaltet
}

\author{
Jeremy Gloor
}

Freier Journalist

\author{
Sinnvolle Gestaltung und subtile Details verhelfen medizinischen Einrichtungen \\ zu mehr Effizienz in Arbeitsabläufen und grösserem Wohlbefinden bei Patienten \\ und Mitarbeitenden. Eine intensive Zusammenarbeit zwischen Ärzten und Archi- \\ tekten schafft dabei statt einfallsloser Umgebung eine logische Szenographie der \\ Räume und ein Umfeld, mit dem sich Kunden wie Arbeitskräfte identifizieren \\ können.
}

Welches sind die entscheidenden gestalterischen Elemente einer zeitgemässen und erfolgreichen Praxis? Es soll darin effizient gearbeitet werden, sie soll dabei ästhetisch vollkommen überzeugen und ein Umfeld bieten, mit dem sich Mitarbeitende wie auch Patienten identifizieren können. Zudem sollen sich Kunden bei jedem Besuch individuell und besonders behandelt fühlen. All diese Aspekte werden zu einer erfolgsversprechenden Klinik führen, in der ansprechende Ästhetik angenehme Arbeitsabläufe unterstützt. Um dahin zu gelangen, gibt es keinen vorgegebenen Prozess. Der Arzt ist der Auftraggeber und hat die Erfahrung, wie er und seine Praxis am besten funktioniert. Er gibt den Inhalt vor. Die Architekten nehmen ihre Rolle als Berater und Planer wahr und bilden für den Inhalt den sinnvollsten Rahmen, der einem gesamtheitlichen Anspruch gerecht wird.

\section{An welchem Punkt in der Praxis befindet sich der Patient in welcher Stimmung?}

Ein Arztbesuch ist schliesslich eine aufregende Angelegenheit. Mit Befürchtungen, Hoffnung und Erleichterung ist er für viele Patienten mit äusserst emotionalen und nervenaufreibenden Momenten verbunden. Allzu oft lassen die architektonischen Umstände von Praxen und Kliniken diese fast gänzlich ausser Acht, und Patienten werden in nüchternen, einfallslosen und gar beklemmenden Warteräumen und Untersuchungszimmern förmlich sitzen gelassen. So wird hierzulande zumindest das Bild einer Grosszahl von medizinischen Einrichtungen gezeichnet. Leider. Und unnötigerweise. In gemeinsamer Erarbeitung mit den jeweiligen Ärzten kann der Praxis oder Klinik, zuverlässig und subtil, ein überzeugendes Ambiente von Vertrauen, Ruhe und Sicherheit verliehen werden.

\section{Wohlgefühl und sinnvolle Arbeitsabläufe}

Dabei gilt es zwei grundlegende Aspekte besonders herauszuarbeiten: Erstens, das Wohlempfinden der Menschen, die sich in der medizinischen Einrichtung aufhalten, also Patienten genauso wie Mitarbeitende. Zweitens, das Kreieren von sinnvollen Arbeitsabläufen, damit Prozesse vereinfacht und die Behandlung von Patienten effizienter gestaltet werden können. Im Fokus stehen dabei die intuitiven Patientenführungen; der gezielte Einsatz von Akustik und Beleuchtung; oder die bewusst gestaltete Begegnung zwischen $\mathrm{Pa}$ tienten und Mitarbeitenden an spezifisch geplanten Orten. Damit sich der Kunde, der zweifelsohne im Zentrum steht, gut aufgehoben fühlt, die unterschwellige Wirkung der Räume ihm ein wohliges Gefühl vermittelt und er nach Verlassen der Praxis deren Besuch als grundsätzlich positives Erlebnis wahrnimmt.

Doch zu Beginn einer Zusammenarbeit steht immer der Auftraggeber und seine persönlichen Vorstellungen. Diese mögen sich in Ausdrücken wie «helle Atmosphäre» oder "viel Holz» äussern, doch viel mehr als diese Begrifflichkeiten direkt umzusetzen, liegt der Anspruch im Gestaltungsprozess darin, das Gefühl festzumachen, welches vermittelt werden soll. Jeder Auftraggeber hat wohl den Anspruch, sich davon abzuheben, was Patienten bisher kannten. Mit Vorstellungen von Helligkeit und Holz soll dabei etwa in der Klinik das Ambiente eines Wohnzimmers entstehen, 
ohne dass an professionellem Auftreten eingebüsst wird. Die Vorstellungen der Bauherrschaft werden also erst einmal in Form einer gesammelten Bilderwelt in Betracht gezogen und von einer ebensolchen Bildersammlung der Architekten erwidert. Es handelt sich dabei um die Phase der gestalterischen Grundbesprechung, in der auch eine Art der Schulung und Sensibilisierung der Bauherren durch die Architekten stattfindet. Man stimmt dabei die erwähnten Bilderwelten auf dieselben Werte ab. Auf diese Richtwerte und Bildwelten, welche die zu vermittelnde Stimmung wiedergeben, kann man sich im Laufe der Arbeit immer beziehen.

Das Optische ist allerdings nicht ausschlaggebend, vielmehr soll in der Planung eine Szenographie der Räume erarbeitet werden, die für angenehme Raumstimmungen sorgt und schliesslich für das positive Praxiserlebnis eines jeden Patienten. Dafür werden als Nächstes die internen Prozesse ins besondere Augenmerk gerückt sowie die essentielle Frage gestellt, welcher Prozess wo stattfinden soll. Arbeitsabläufe können und sollen dabei in Frage gestellt und womöglich Lösungen zu deren Verbesserung gefunden werden. An welcher Position in der Praxis geht es um die Vermittlung welcher Information und welche Stimmung soll dabei vorherrschen? Der Arzt und Bauherr ist hier der Fachmann. Architekten stellen Fragen und erbringen Vorschläge. Es braucht dafür eine ehrliche, direkte und intensive Phase der Gespräche und des Aufbaus von Vertrauen. Die Beantwortung zahlreicher Fragen ergibt schliesslich die Aufteilung der Räume nach einer klaren Logik. Erst danach kümmert man sich um die eigentliche Einrichtung. Das Selbstverständnis einer diesbezüglich ansprechenden Lösung macht die Oberflächen (und damit die verwendeten Materialien) zweitrangig und bietet die Möglichkeit, einen vorgegebenen Kostenrahmen einzuhalten.

Die Kreation der sinnvollen Szenographie wird auch mit dem Spiel der Proportionen von Räumlichkeiten erlangt, mit dem gezielten Einsatz von Enge und Weite, Hell und Dunkel, sowie dem Schaffen von Plätzen innerhalb einer in sich geschlossenen Praxis. Das heisst, Warteräume, Untersuchungs- und Ärztezimmer erhalten unterschiedliche Grössen, die je nach Zweck oder Erfüllung eines Prozesses bestimmt werden. Braucht es etwa Platz für Untersuchungsgeräte oder sollen sich Patienten besonders geborgen fühlen? Sollen sie im Warteraum, den sie nach der Untersuchung betreten, das Gefühl erhalten, richtig durchatmen zu können? Also grundsätzlich gefragt: An welchem Punkt in der Praxis befindet sich der Patient in welcher Stimmung? Und soll diese unterstützt oder abgeschwächt werden?

\section{Intuitive Patientenführung durch leitende Innenarchitektur}

Grundsätzlich sollen in einer medizinischen Einrichtung durch sinnvolle Gestaltung und reibungslose $\mathrm{Ab}$ läufe das Gefühl von Ruhe und Geborgenheit und dahingehend das Vertrauen in das Personal gefördert werden. Patienten wollen sich sicher fühlen. Um dies $\mathrm{zu}$ erreichen, ist mitunter ausschlaggebend, wie viel Zeit sich das Personal an welcher Position in der Praxis für den Kunden nimmt. Am Empfang zum Beispiel: Erhält der Patient hier ein Formular auf einem Klappbrett in die Hand gedrückt, oder setzt man sich an einen Tisch, und die Personalien werden von einem Mitarbeitenden aufgenommen? Letzteres vermittelt zweifelsohne ein Gefühl von ernst gemeinter Aufmerksamkeit. Dasselbe gilt bei der Verabschiedung, wenige Worte und nur einige Sekunden an persönlichem Aufwand können an gezielten Stellen einen eindrücklichen Effekt haben. Geplant wird hinsichtlich dieses Effekts bis ins Detail und zum Beispiel dem Stuhl, auf dem der Patient bei der Registrierung sitzt. Steht hier ein Hocker oder ein Stuhl mit Armlehne? Hat er Rollen oder kann er sich gar drehen? Was für ein Gefühl vermittelt, wohl unbewusst, die Wahl des Stuhlmodells?

\section{Es gilt auch, Begegnungen zwischen Patienten und Mitarbeitenden gezielt zu steuern.}

Ein weiterer Aspekt, der durch smarte Architektur auf subtile Weise herbeigeführt wird, ist die intuitive Patientenführung. Dies bedeutet, dass Patienten in einer Klinik vorwiegend ohne Beschilderung ihren Weg von der einen Räumlichkeit zur nächsten finden. Dies kann durch den Einsatz von prägnanten Materialien entstehen, welche eine Leitlinie bilden, oder starke Orientierungspunkte wie Pflanzen oder Kunstwerke. So ist oft keine Begleitperson notwendig, und Patienten fühlen sich dennoch nicht verloren. Durch kurze Wege kann zudem Zeit eingespart werden. Es ist eine weitere Möglichkeit, eine effiziente Praxis zu schaffen und Kosten an dafür angebrachten Stellen einzusparen.

Erwähnenswert ist an dieser Stelle auch die Wichtigkeit von zwei oder mehr Wartebereichen, mindestens einen vor und einen nach der Erstuntersuchung, denn Patienten sind in verschiedenen Stimmungen und würden unter Umständen im selben Raum für gegenseitige Unruhe sorgen. Diese Stimmungen können in den unterschiedlichen Bereichen abgefangen werden. Zudem entsteht durch den neuen Warteraum das Gefühl, dass es mit dem Prozess vorwärtsgeht. 
Es gilt auch, Begegnungen zwischen Patienten und Mitarbeitenden gezielt zu steuern. Dazu braucht es grundsätzlich zwei Eingänge, einen für Patienten und einen separaten für Mitarbeiter und Ärzte. Sollten Letztere zum Beispiel verspätet von einem Notfall in der Praxis eintreffen, können sie diskret eintreten, und es kommt bei Patienten keinesfalls dadurch bedingte Nervosität auf. Auch ein abgeschirmter Arbeitsbereich für das Personal ist wichtig, um darin einen geschützten internen Begebungsort zu schaffen, in dem unbehelligt geredet werden kann und gewisse Gesprächsthemen nicht für Irritation bei Patienten sorgen. Ein schalldichter Ort also, in dem nicht geflüstert werden muss, was Patienten wiederum verunsichern könnte. Dieser Ort soll es Mitarbeitenden auch ermöglichen, schnell und nicht vor den Augen der Patienten von Raum A zu Raum B zu gelangen.

Jeremy Gloor

c/o Dost Innenarchitektur

Finsterwaldstr. 109

CH-8200 Schaffhausen

www.dost.org
All dies schafft ein unverkrampftes Miteinander von Ärzten, Patienten und Mitarbeitern. Alle fühlen sich frei, ungezwungen, müssen sich nicht verstellen und fühlen ihre Privatsphäre respektiert und gewahrt. Ein reibungsloser Arbeitsablauf durch ein Netzwerk mit einwandfreiem Patientenfluss ist das Ziel, welches mit gestalterischen Werten erreicht wird. Diese sind von blossem Auge nicht zwingend zu erkennen. Das zeichnet die angemessene und erfolgreiche Architektur einer Praxis oder Klinik aus. Sie schafft bewusste Begegnungen und schafft das Vertrauen ineinander. Sie kreiert eine Umgebung, die es erlaubt, dass im fliessenden Prozess die menschlichen Kontakte nach wie vor elementar sind. Dabei erzählt sie Geschichten, welche zum guten Gefühl der Patienten beitragen, das sie auf dem Heimweg mit sich tragen, und hat damit einen unbestreitbaren Wert.

Disclosure statement

Der Artikel wurde im Auftrag von Dost Innenarchitektur erstellt. 